\title{
CHOLANGIOHEPATITIS IN BROILER CHICKENS IN JAPAN: HISTOPATHOLOGICAL, IMMUNOHISTOCHEMICAL AND MICROBIOLOGICAL STUDIES OF SPONTANEOUS DISEASE
}

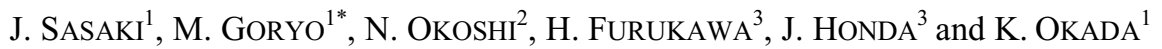 \\ ${ }^{1}$ Department of Veterinary Pathology, Faculty of Agriculture, Iwate University, Morioka, \\ Iwate 020-8550; ${ }^{2}$ Fukushima Kenhoku Institute of Public Health, Fukushima, Fukushima \\ 960-8132; ${ }^{3}$ Meat Inspection Center of Fukushima Prefecture, Fukushima, \\ Fukushima 960-0101, Japan
}

(Received April 19, 1999; accepted June 24, 1999)

\begin{abstract}
Forty-five broiler carcasses from 6 different flocks were condemned due to liver lesions at processing meat inspection, and collected for pathological and bacterial examinations. All affected chickens showed liver enlargement with discolouration and an apparent acinar pattern. The enlarged gallbladder and the extrahepatic bile ducts contained yellow inspissated cream-coloured material. Histopathologically, extensive proliferation of bile ductules with fibrosis was observed in interlobular connective tissue, and it spread to form bridges with adjoining triads. Destruction and obstruction of portal bile ducts with multiple granulomas due to bacterial infection and outflow of the bile were frequently observed. Many Gram-positive bacilli were seen in the lesions, and they were identified as Clostridium perfringens by indirect immunofluorescence staining technique. Clostridium perfringens was isolated from affected livers. These findings are consistent with cholangiohepatitis. Therefore, it is suggested that $C$. perfringens might be important in the pathogenesis of cholangiohepatitis in broiler chickens.
\end{abstract}

Key words: Broiler chicken, cholangiohepatitis, Clostridium perfringens, multiple granuloma, proliferation of bile ductules

Liver abnormality in broiler carcasses characterized by enlarged, pale, firm livers, associated with thickened gallbladder walls and bile ducts distended with bile, was first described in Scotland (Randall et al., 1983). Since then, a similar condition has also been reported in Scotland (Randall et al., 1986), in Canada as hepatosis (Hutchison and Riddell, 1990) and as fibrosing cholehepatitis (Onderka et al., 1990). The disease has been recently recognised as cholangiohepatitis (Randall, 1991, 1996).

*Corresponding author; E-mail: goryo@iwate-u.ac.jp; Fax: 81-019-621-6231 
Histopathologically, extensive proliferation of bile ductules with fibrosis and multiple granulomas are characteristic features (Randall et al., 1983, 1986; Hutchison and Riddell, 1990; Onderka et al., 1990; Mabuchi et al., 1996; Sasaki et al., 1997, 1998). Although the causative agent is unknown, Clostridium perfringens has been isolated from the abnormal livers, gallbladders and extrah epatic bile ducts of affected chickens (Randall et al., 1983, 1986; Hutchison and Riddell, 1990; Sasaki et al., 1997, 1998). Most of the livers and biliary tracts e xamined have presented Gram-positive bacilli by histopathological examination (Mabuchi et al., 1996; Sasaki et al., 1997, 1998). On the other hand, Escherichia coli, Pasteurella haemolytica, Streptococcus equisimilis, Campylobacter sp. and adenovirus have also been isolated from affected livers and bile (Randall et al., 1983, 1986; Hutchison and Riddell, 1990).

This paper describes the pathological and bacteriological aspects of cholangiohepatitis associated with $C$. perfringens in broiler chickens.

\section{Materials and methods}

\section{Chickens}

Forty-five broiler carcasses from six different flocks were condemned for liver lesions in a commercial broiler farm at the Fukushima Meat Inspection Center. The bird tissues were collected for histopathological, immunohistochemical and bacteriological examinations at meat inspection. Healthy broiler chickens from the same flocks were also collected as controls.

\section{Histopathology}

Following postmortem examination, tissue samples from the liver, spleen, kidney, heart, lung, pancreas, duodenum, jejunum, ileum and caecal tonsil were collected, fixed in 10\% formalin, processed routinely, and embedded in paraffin wax. Sections were cut approximately $4 \mu \mathrm{m}$ thick and stained with haematoxylin and eosin (HE). Liver sections were also stained with Masson trichrome, reticulin silver impregnation and Gram's stain.

\section{Immunohistochemistry}

Clostridium perfringens was identified by an indirect immunofluorescence staining technique using sections of the liver, gallbladder and extrahepatic bile ducts. Paraffin-embedded sections of tissue samples were deparaffinized, rehydrated, and rinsed in phosphate buffered saline (PBS), pH 7.2. Specimens were incubated overnight at $4{ }^{\circ} \mathrm{C}$ with a chicken polyclonal anti- $C$. perfringens type A serum prepared against the $C$. perfringens isolated from affected liver (Sasaki et al., 1997). The primary antibody of anti- $C$. perfringens serum was applied at 
1:100 dilution, though positive reactivity to the isolate of $C$. perfringens was confirmed at 1:2000 dilution of antiserum. Chicken polyclonal anti-Escherichia coli, anti-Staphylococcus aureus sera and normal chicken serum were used as controls. A fluorescein isothiocyanate-conjugated anti-chicken rabbit $\operatorname{IgG}$ (Chemicon International INC., USA) was used and the sections were incubated for $30 \mathrm{~min}$ at $37{ }^{\circ} \mathrm{C}$. Unbound conjugate was removed by rinsing with three changes of PBS. Finally, the sections were examined by ultraviolet microscopy.

\section{Bacteriology}

The affected livers of chickens in Groups A and F were cultured aerobically and anaerobically. The specimens were stamped onto blood agar or Clostridium welchii $(\mathrm{CW})$ agar base-without kanamycin (Nissui Co., Japan) and incubated at $37^{\circ} \mathrm{C}$ for $24 \mathrm{~h}$ in a catalytic anaerobic jar using a Gas Pak hydrogen generator (Difco Laboratories, Detroit, USA). Organisms were identified by standard tec hniques (Sasaki et al., 1997).

\section{Results}

\section{Gross pathology}

The birds were 51 to 56 days of age and had shown no clinical signs or elevated mortality rates, though growth retardation and emaciation were noted. Gross lesions were detected in the liver, gallbladder and extrahepatic bile ducts. The livers were enlarged (Table 1) with discolouration and an apparent acinar pattern in most cases, and often showed small white, green or brown foci beneath the capsule and throughout the parenchyma (Fig. 1). The gallbladders and extr ahepatic bile ducts were thickened and distended with viscous bile or inspissated cream-coloured material in some cases. Fresh patchy haemorrhages of intestine, especially the caecum with dark red-coloured material in the lumen, were frequently observed in flock F. No gross lesions were found in other organs.

Table 1

Liver, spleen and carcass weights in the affected chickens

\begin{tabular}{lccc}
\hline \multicolumn{1}{c}{ Flock } & Liver $(\mathrm{g})$ & Spleen $(\mathrm{g})$ & Carcass $(\mathrm{kg})$ \\
\hline $\mathrm{A}(\mathrm{n}=5)$ & $135.0 \pm 25.1^{*}$ & $5.2 \pm 1.4$ & $1.8 \pm 0.5$ \\
$\mathrm{~B}(\mathrm{n}=3)$ & $\mathrm{NE}$ & $\mathrm{NE}$ & $\mathrm{NE}$ \\
$\mathrm{C}(\mathrm{n}=9)$ & $99.6 \pm 16.6$ & $5.0 \pm 1.8$ & $2.0 \pm 0.2$ \\
$\mathrm{D}(\mathrm{n}=3)$ & $159.1 \pm 31.5$ & $3.3 \pm 0.9$ & $\mathrm{NE}$ \\
$\mathrm{E}(\mathrm{n}=5)$ & $\mathrm{NE}$ & $\mathrm{NE}$ & $\mathrm{NE}$ \\
$\mathrm{F}(\mathrm{n}=19)$ & $45.1 \pm 10$ & $\mathrm{NE}$ & $\mathrm{NE}$ \\
\hline
\end{tabular}

${ }^{*}$ Mean $\pm \mathrm{SD} ; \mathrm{NE}=$ not examined 


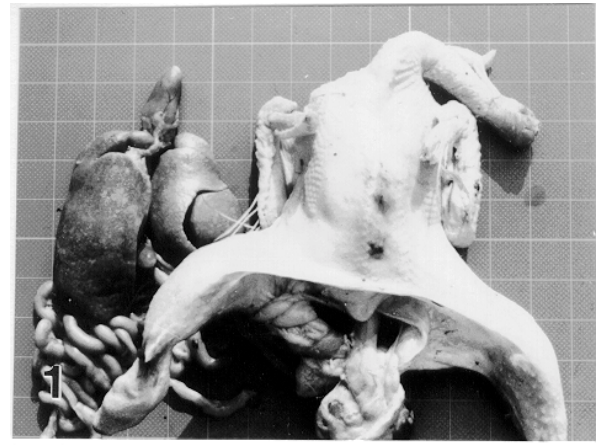

Fig. 1. Liver enlargement and acinar pattern are obvious in an affected carcass

\section{Histopathology}

Most of the livers examined showed a similar histopathological picture of a chronic and fibrosing hepatitis (Table 2). The principal change was prolifer ation of the bile ductules with fibrosis and multiple granulomas. Proliferation of the bile ductules started periportally and spread to form bridges with adjoining triads (Fig. 2). The lesions in the livers revealed various stages. Hepatocytes were compressed by the proliferation of bile ductules, which eventually replaced most of the normal parenchyma. Proliferative bile ductules had cuboidal or columnar epithelial cells with a round nucleus and pale-staining cytoplasm (Fig. 3). These ducts were surrounded by a delicate network of reticular fibres.

Multiple granulomas due to outflow of the bile and/or bacterial infection were observed in the intrahepatic bile ducts (Fig. 4). Intrahepatic cholangitis was variable but there was usually some evidence of biliary stasis and bacterial i nfection. The lumen of portal bile ducts contained purulent material and Grampositive bacilli, and there was periductal fibrosis and infiltration of inflammatory cells (Fig. 5). Lympho-plasmacytic accumulation with germinal centres was fr equently seen around such lesions. Immature myelocytic granulocytes were also seen in the portal areas. Frequent mitoses among these granulocytes were an i ndication of extramedullary haematopoiesis characterized by the presence of myelocytes.

The mucosa of the gallbladder was necrotic and thickened as a result of i nfiltration of granulocytes in severe cases. Chronic inflammation consisted of i nfiltration of lymphocytes and plasma cells (Fig. 6). Extrahepatic bile ducts were distended, and similar lesions were observed in the gallbladder.

Many Gram-positive bacilli were seen in the intrahepatic bile ducts, granulomas, gallbladder and extrahepatic bile ducts accompanied by inflamm atory lesions (Fig. 7). Histopathologically, no evidence of viral infection was seen in any case. 

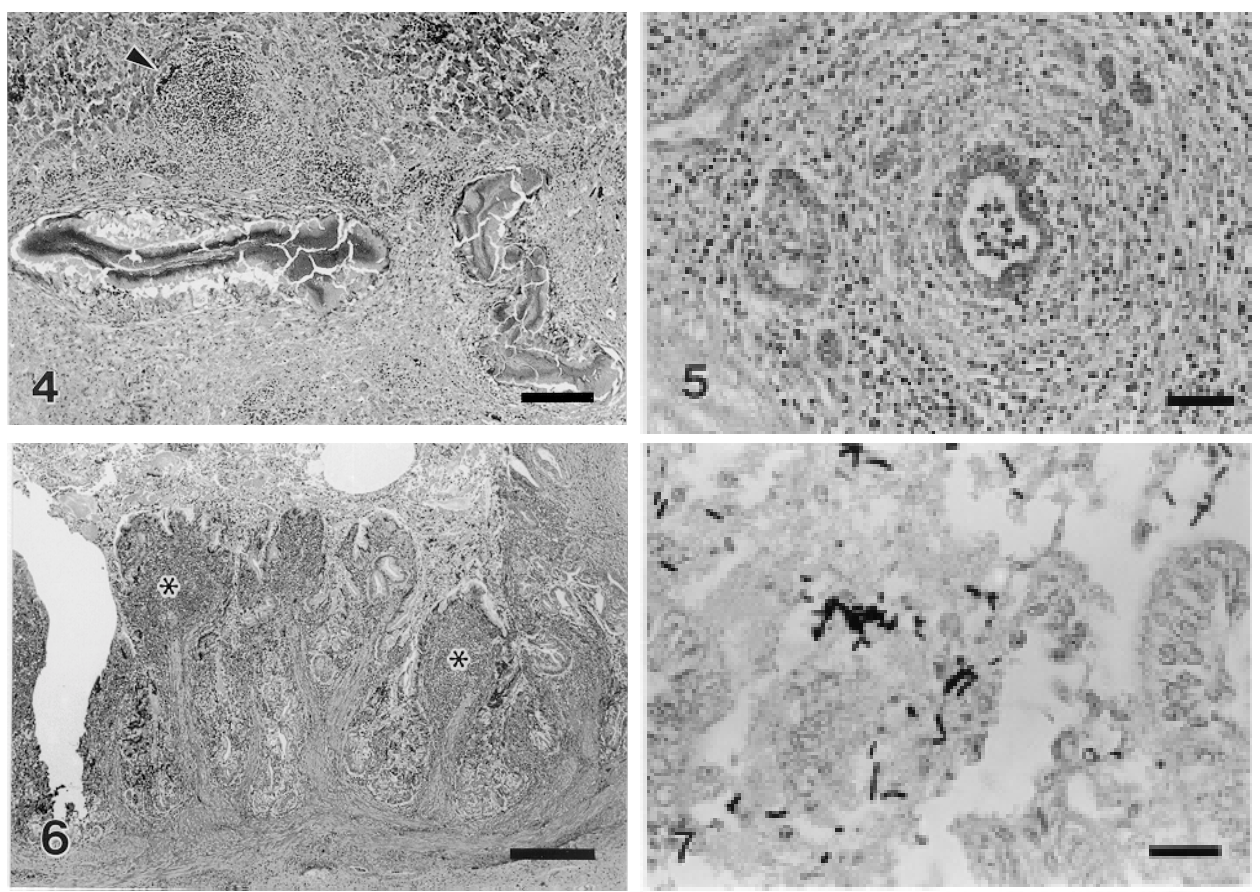

Fig. 4. Multiple granulomas due to outflow of the bile in the intrahepatic bile duct. Lymphoid accumulation is seen (arrowhead). HE, Bar $=200 \mu \mathrm{m}$

Fig. 5. Cholangitis associated with periductal fibrosis in the portal area. Lymphocytic and heterophilic infiltration is seen. HE, Bar $=50 \mu \mathrm{m}$

Fig. 6. Chronic cholecystitis. Infiltration of lymphocytes and plasma cells in the lamina propria (asterisks). HE, Bar $=300 \mu \mathrm{m}$

Fig. 7. Cholangitis associated with Gram-positive bacilli in the extrahepatic bile duct. Gram's stain, $\mathrm{Bar}=20 \mu \mathrm{m}$

Severe to mild coccidiosis was observed in the duodenum, ileum and ca ecum in fifteen cases of flock F.

\section{Immunohistochemistry}

By an indirect immunofluorescence staining technique, many rod-shaped bacilli reacted positively with anti- $C$. perfringens type A sera in intrahepatic bile ducts, granulomas, gallbladders and extrahepatic bile ducts (Fig. 8).

\section{Microbiology}

Clostridium perfringens was isolated anaerobically in pure culture from 3 out of 5 (flock A) and 4 out of 4 (flock F) affected livers (Table 3). The bact erium was identified by its characteristic stormy fermentation reaction in litmus 
milk and the production of acid from glucose, lactose and sucrose. Alpha toxin was produced, as was shown by lecithinase activity on $\mathrm{CW}$ agar. Using the $C$. perfringens differentiation strip (Nissui Co., Japan), inhibition of lecithinase activity was observed around the strip. E. coli and Streptococcus sp. were also isolated aerobically from affected livers.

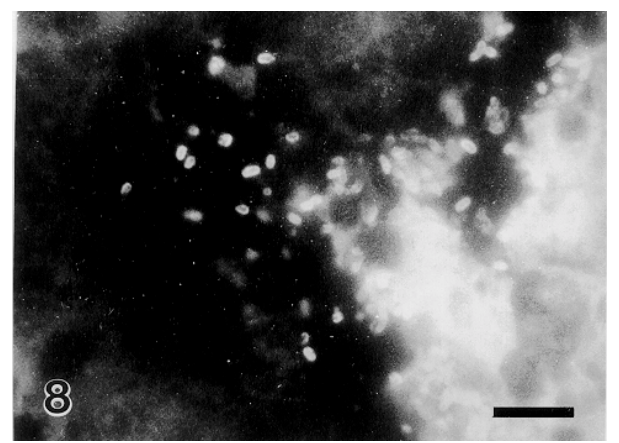

Fig. 8. Cholangitis of the intrahepatic bile duct. Many rod-shaped bacilli are strongly positive to a polyclonal chicken antibody raised against $C$. perfringens by an indirect immunofluorescence staining technique. $\mathrm{Bar}=20 \mu \mathrm{m}$

Table 3

Results of anaerobic and aerobic bacterial examination of affected livers

\begin{tabular}{lccc}
\hline Flock & No. & Anaerobic culture & Aerobic culture \\
\hline $\mathrm{A}$ & 1 & $+^{*}$ & $\mathrm{NE}$ \\
& 2 & + & $\mathrm{NE}$ \\
& 3 & - & $\mathrm{NE}$ \\
& 4 & + & $\mathrm{NE}$ \\
\hline $\mathrm{F}$ & 5 & + & E. coli / Streptococcus $\mathrm{sp}$. \\
& 2 & + & E. coli \\
& 5 & + & E. coli / Streptococcus $\mathrm{sp}$. \\
& 12 & + & E. coli / Streptococcus $\mathrm{sp}$. \\
\hline
\end{tabular}

${ }^{*}$. perfringens $:+=$ isolation; $-=$ no isolation; $\mathrm{NE}=$ not examined

\section{Discussion}

The gross lesions in the present cases were characterized by an enlarged, firm, tan-coloured liver with an apparent acinar pattern. Histopathologically, proliferation of bile ductules accompanied by fibrosis, multiple granulomas of the 
liver, chronic cholecystitis and cholangitis were observed. These observations were consistent with cholangiohepatitis in broiler chickens (Randall, 1991, 1996).

Randall $(1991,1996)$ described that Gram-positive bacilli were often o bserved in lesions and $C$. perfringens was isolated on anaerobic bacterial culture from affected liver and gallbladder. Onderka et al. (1990) reported that fibrosing cholehepatitis with proliferation of bile ductules was reproduced by inoculation of $C$. perfringens into the hepatoenteric bile duct after bile duct ligation. Hist opathologically, Gram-positive bacilli were present in 40 out of 45 affected livers, 35 out of 42 gallbladders, and 30 out of 40 extrahepatic bile ducts. The isolation of $C$. perfringens from the affected livers also supports the histological findings. Our study was in accordance with previous investigations of spontaneous and experimental cholangiohepatitis, indicating that $C$. perfringens plays a role in the pathogenesis of cholangiohepatitis.

It has been suggested that the infection route of the disease may be ha ematogenous, lymphogenous or by ascending bacterial infection from the intestine (Kelly, 1993; Mabuchi et al., 1996; Sasaki et al., 1997, 1998). The present study supports ascending bacterial infection because principal lesions were restricted to the hepatobiliary tracts.

Cholangiohepatitis in broiler chickens has been reported sporadically, and a small number of affected chickens per flock are usually detected at slaughter (Mabuchi et al., 1996; Sasaki et al., 1997, 1998). Collective outbreaks of cholangiohepatitis have never been reported, but twenty cases in flock $\mathrm{F}$ were from a flock of 2,542 birds $(0.79 \%$ morbidity). This incidence was considered a colle ctive outbreak of cholangiohepatitis, and suggested that the disease is not a sp oradic disease.

Extensive proliferation of bile ductules is the most characteristic feature of cholangiohepatitis in broiler chickens. These lesions tend to start periportally, and spread to form bridges with adjoining triads. Slight to severe degrees of these lesions were seen in this study, and were correlative with cholestasis. It is suggested that the degree of proliferation of bile ductules reflects an affective stage of cholangiohepatitis.

Clostridium perfringens may play an important role in cholangiohepatitis of the broiler chicken as described in previous reports (Hutchison and Riddell, 1990; Onderka et al., 1990; Randall, 1991, 1996) and as found in our investig ations (Sasaki et al., 1997, 1998). However, this bacterium usually forms part of the normal intestinal flora of man and animals and can be found in the soil (Nillo, 1980). Coccidiosis has marked influence on the intestinal flora of chic kens (Bradley and Radhakrishnan, 1973), and may predispose to overgrowth of $C$. perfringens in the intestine. Coccidiosis was found in the present study, but the relationship between $C$. perfringens and coccidiosis was unknown. Cholangiohepatitis was not reproduced in chicks inoculated orally with a $C$. perfringens isolate from affected liver in our previous experimental study (Sasaki et al., 
1997). Further studies are needed to establish the association of cholangiohepatitis due to C. perfringens in broiler chicken.

\section{References}

Bradley, R. E. and Radhakrishnan, C. V. (1973): Coccidiosis in chickens: Obligate relationship between Eimeria tenella and certain species of cecal microflora in the pathogenesis of the disease. Avian Dis. 17, 461-476.

Hutchison, T. W. S. and Riddell, C. (1990): A study of hepatic lesions in broiler chickens at processing plants in Saskatchewan. Can. Vet. J. 31, 20-25.

Kelly, W. R. (1993): Liver and biliary system. In: Jubb, K. V. F., Kennedy, P. C. and Palmer, N. (eds) Pathology of Domestic Animals, Vol. 2, $4^{\text {th }}$ edn. Academic Press Inc., San Diego, pp. 319-406.

Mabuchi, K., Goryo, M., Miura, S., Okoshi, N. and Okada, K. (1996): Pathology of cholangiohepatitis in broiler chickens (in Japanese, with English abstract). J. Jpn. Soc. Poult. Dis. 32, $84-89$.

Nillo, L. (1980): Clostridium perfringens in animal disease: A review of current knowledge. Can. Vet. J. 21, 141-148.

Onderka, D. K., Langevin, C. C. and Hanson, J. A. (1990): Fibrosing cholehepatitis in broiler chickens induced by bile duct ligations or inoculation of Clostridium perfringens. Can. J. Vet. Res. 54, 285-290.

Randall, C. J. (1991): Cholangiohepatitis in broilers. In: Disease \& Disorder of the Domestic Fowl $\&$ Turkey, $2^{\text {nd }}$ edn. Wolfe Publishing Ltd., London. pp. 134-136.

Randall, C. J. (1996): Cholangiohepatitis. In: Colour Atlas of Avian Histopathology. Wolf Publishing Ltd., London. pp. 90-92.

Randall, C. J., Kirkpatrick, K. S. and Pearson, D. B. (1986): Liver abnormality in broilers. Vet. Rec. 119, 576.

Randall, C. J., Stevens, H., Walsby, J. B. and Ashton, W. L. (1983): Liver abnormality in broiler carcases. Vet. Rec. 112, 159

Sasaki, J., Goryo, M., Furukawa, H., Okoshi, N and Okada, K. (1997): Pathology of cholangiohepatitis in broiler chickens, isolation and identification of Clostridium perfringens from affected livers, and experimental study in chicks inoculated with $C$. perfringens (in Japanese, with English abstract). J. Jpn. Soc. Poult. Dis. 33, 79-85.

Sasaki, J., Goryo, M., Furukawa, H., Okoshi, N. and Okada, K. (1998): An outbreak of cholangiohepatitis due to Clostridium perfringens in broiler chickens (in Japanese, with English abstract). J. Jpn. Vet. Med. Assoc. 51, 528-532. 